PROCEEDINGS OF THE

AMERICAN MATHEMATICAL SOCIETY

Volume 128, Number 7, Pages 2069-2074

S 0002-9939(99)05257-0

Article electronically published on October 29, 1999

\title{
SPECTRAL STRUCTURE AND SUBDECOMPOSABILITY OF $p$-HYPONORMAL OPERATORS
}

\author{
RUAN YINGBIN AND YAN ZIKUN
}

(Communicated by David R. Larson)

\begin{abstract}
We prove that for every $p$-hyponormal operator $A, 0<p \leq 1$, there corresponds a hyponormal operator $\tilde{A}$ such that $A$ and $\tilde{A}$ have "equal spectral structure". We also prove that every $p$-hyponormal operator $A, 0<p \leq 1$, is subdecomposable. Then some relevant quasisimilarity results are obtained, including that two quasisimilar $p$-hyponormal operators have equal essential spectra.
\end{abstract}

\section{INTRODUCTION AND NOTATION}

Let $\mathbf{H}$ be a complex separable Hilbert space and let $L(\mathbf{H})$ denote the algebra of all bounded linear operators on $\mathbf{H}$. An operator $A \in L(\mathbf{H})$ is said to be $p$-hyponormal, $0<p \leq 1$, denoted as $A \in p$ - $\mathbf{H}$, if $\left(A A^{*}\right)^{p} \leq\left(A^{*} A\right)^{p}$. An 1-hyponormal operator is hyponormal, and a $\frac{1}{2}$-hyponormal operator is said to be semi-hyponormal. In the sequel, for every $A \in L(\mathbf{H})$, we define $\hat{A}$ by $\hat{A}=|A|^{\frac{1}{2}} U|A|^{\frac{1}{2}}$ where $U,|A|$ are as in the polar decomposition $A=U|A|$. Let $\hat{A}$ have the polar decomposition $\hat{A}=V|\hat{A}|$. The operator $\tilde{A}$ is then defined by $\tilde{A}=|\hat{A}|^{\frac{1}{2}} V|\hat{A}|^{\frac{1}{2}}$. Aluthge [1] showed that for $A \in p-\mathbf{H}, 0<p \leq 1, \hat{A}$ is semi-hyponormal and $\tilde{A}$ is hyponormal. Some authors paid attention to the relations between the spectral structure of $A$ and $\hat{A}$ (e.g. [2, 3], 4]). In this note, we prove that for general $A \in p-\mathbf{H}, 0<p \leq 1, A, \hat{A}$ and $\tilde{A}$ have "equal spectral structure", i.e. $\sigma_{s}(A)=\sigma_{s}(\hat{A})=\sigma_{s}(\tilde{A})$, where $\sigma_{s}=$ $\sigma, \sigma_{a}, \sigma_{r}, \sigma_{B}, \sigma_{w}, \sigma_{e}, \sigma_{k}, \sigma_{D}, \psi_{n}, \psi_{m n}$ or $\sigma_{p}^{0}$.

A subdecomposable operator is, up to similarity, the restriction of a decomposable operator to its invariant space. J. Eschmeier [5] proved that $A \in L(\mathbf{H})$ is subdecomposable if and only if $A \in(\beta)$, i.e. $A$ has Bishop's property ( $\beta)$. M.Putinar and J.Eschmeier [6, 7] proved that hyponormal operators are subscalar and therefore subdecomposable. B.Duggal [2] asked whether a general $p$-hyponormal operator satisfies condition $(\beta)$. We give an affirmative answer to this question. Yang Liming 8] proved that two quasisimilar hyponormal operators have equal essential spectra. By means of the subdecomposability of $A \in p-\mathbf{H}$, we generalize and strengthen this result to general $p$-hyponormal operators $(0<p \leq 1)$.

For $T \in L(\mathbf{H}), \sigma(T), \sigma_{p}(T), \sigma_{a}(T)$, and $\sigma_{e}(T)$ denote the spectrum, point spectrum, approximate point spectrum and essential spectrum of $T$, respectively. Write

Received by the editors August 27, 1998.

1991 Mathematics Subject Classification. Primary 47B99, 47A10.

Key words and phrases. Spectra, subdecomposability, p-hyponormal, quasisimilarity.

This research was supported by the National Natural Science Foundation of China. 
$\nu(T)=\operatorname{dim} \operatorname{Ker} T, \quad \mu(T)=\operatorname{dim} \operatorname{Ker} T^{*}$; the index of $T$ is defined by $\operatorname{ind} T=$ $\nu(T)-\mu(T)$ if at least one of $\nu(T)$ and $\mu(T)$ is finite. Let $\psi$ denote the set of all semi-Fredholm operators on $\mathbf{H}$. Write $\rho_{s-F}(T)=\{\lambda \in \mathbf{C}: T-\lambda \in \psi\}, \psi_{n}(T)=$ $\{\lambda \in \mathbf{C}: R(T-\lambda)$ is closed, ind $(T-\lambda)=n\}(n=0, \pm 1, \pm 2, \cdots, \pm \infty), \psi_{m n}(T)=$ $\{\lambda \in \mathbf{C}: R(T-\lambda)$ is closed, $\nu(T-\lambda)=m, \mu(T-\lambda)=n\}(m, n=0,1,2, \cdots, \infty)$. Also write $\psi_{+}(T)=\bigcup_{1<n<\infty} \psi_{n}(T), \quad \psi_{-}(T)=\bigcup_{1<n<\infty} \psi_{-n}(T), \sigma_{r}(T)=\{\lambda \in$ C : $\operatorname{ker}(T-\lambda)=\{0\}, \bar{R}(\bar{T}-\lambda)$ is closed, $R(T-\bar{\lambda}) \neq \mathbf{H}\}, \sigma_{D}(T)=\{\lambda \in \mathbf{C}:$ $R(T-\lambda)$ is not closed $\}, \sigma_{k}(T)=\mathbf{C} \backslash \rho_{s-F}(T) . \sigma_{p}^{0}(T)$ denotes the set of all isolated eigenvalues of $T$ with finite algebraic multiplicity. $\mathbf{K}(\mathbf{H})$ denotes the set of all compact operators on $\mathbf{H} . \quad \sigma_{B}(T)=\bigcap_{K \in=T K} \in(\mathbf{K})(T+K)=\sigma(T) \backslash \sigma_{p}^{0}(T), \sigma_{w}(T)=$ $\bigcap_{K \in \mathbf{K}(\mathbf{H})} \sigma(T+K)=\sigma(T) \backslash \psi_{0}(T)$.

Suppose $\lambda \in \mathbf{C}, T \in L(\mathbf{H})$. $\lambda$ is called a regular point of the operator $T$ if $\left\|P_{\operatorname{Ker}(T-\mu)}-P_{\operatorname{Ker}(T-\lambda)}\right\| \rightarrow 0(\mu \rightarrow \lambda)$, where $P_{\operatorname{Ker}(T-\lambda)}$ denotes the orthogonal projection onto $\operatorname{Ker}(T-\lambda), \tau^{r}(T)$ denotes the set of all regular points of $T, \tau^{s}(T)=$ $\mathbf{C} \backslash \tau^{r}(T)$. For every set-valued function $B(\cdot): L(\mathbf{H}) \rightarrow 2^{\mathbf{C}}$, write $B^{r}(T)=B(T) \cap$ $\tau^{r}(T), B^{s}(T)=B(T) \cap \tau^{s}(T)$ (see $[9]$ ).

Let $T \in L(\mathbf{H})$. Suppose that the closed subspace $M$ of $\mathbf{H}$ reduces $T$; then $M$ is said to be a normal subspace of $T$ if $\left.T\right|_{M}$ is a normal operator. The operator $T$ is said to be pure if $T$ has no non-trival normal subspace.

$\mathcal{O}(\Omega, \mathbf{H})$ denotes the Fréchet space of all $\mathbf{H}$-valued analytic functions on the open set $\Omega \subset \mathbf{C}$ with the topology defined by uniform convergence on every compact subset of $\Omega$. Suppose $T \in L(\mathbf{H}) ; T$ is said to have Bishop's property $(\beta)$ (denoted by $T \in(\beta))$ if the mapping $\alpha_{T, \Omega}: \mathcal{O}(\Omega, \mathbf{H}) \rightarrow \mathcal{O}(\Omega, \mathbf{H}), f \mapsto(T-z) f$ is injective and has closed range for every open subset $\Omega$ of $\mathbf{C}$. Write $E_{2}(T)=\{\lambda \in \mathbf{C}$ : $\exists \delta>0$ such that for $\Omega=O\left(\lambda, \delta^{\prime}\right), 0<\delta^{\prime}<\delta, \alpha_{T, \Omega}$ has closed range $\}, A(T)=$ $\left\{\lambda \in \mathbf{C}: \exists \delta>0\right.$ such that for $\Omega=O\left(\lambda, \delta^{\prime}\right), 0<\delta^{\prime}<\delta, \alpha_{T, \Omega}$ is injective $\}$. Write $T \in\left(E_{2}\right)((A))$ if for every $\lambda \in \mathbf{C}, \lambda \in E_{2}(T)(A(T))$. $T \in(A)$ is equivalent to $T$ has the single-valued extension property. It is clear by definitions (see 10, Proposition 4]) that $T \in(\beta)$ if and only if $T \in(A)$ and $T \in\left(E_{2}\right)$.

Let $T_{1}, T_{2} \in L(\mathbf{H})$; we say $T_{1}$ is a dense (quasiaffine) transform of $T_{2}$, denoted as $T_{1} \stackrel{d r}{\longrightarrow} T_{2}\left(T_{1} \stackrel{q}{\rightarrow} T_{2}\right)$, if there exists operator $X$ with dense range (injective and dense range) such that $X T_{1}=T_{2} X$. We said $T_{1}$ and $T_{2}$ are densely (quasi-) similar, denoted as $T_{1} \stackrel{d r}{\sim} T_{2}\left(T_{1} \stackrel{q}{\sim} T_{2}\right)$, if $T_{1} \stackrel{d r}{\longrightarrow} T_{2} \stackrel{d r}{\longrightarrow} T_{1}\left(T_{1} \stackrel{q}{\rightarrow} T_{2} \stackrel{q}{\rightarrow} T_{1}\right)$.

\section{Spectral structure of $A$ and $\hat{A}$}

Lemma 1. If $T$ is a pure p-hyponormal operator, then $\sigma_{p}(T)=\emptyset$.

Proof. Let $T$ have the polar decomposition $T=U|T|$. If $\lambda \in \sigma_{p}(T)$, then $\operatorname{Ker}(T-\lambda) \neq\{0\}$. By [11, Proof of Theorem 4], $\operatorname{Ker}(T-\lambda) \subset \operatorname{Ker}(T-\lambda)^{*}$. This implies that $\operatorname{Ker}(T-\lambda)$ is a reducing subspace of $T$ and $\left.T\right|_{\operatorname{Ker}(T-\lambda)}$ is normal, a contradiction to the purity of $T$. Hence $\sigma_{p}(T)=\emptyset$.

Lemma 2. Let $S, T \in L(\mathbf{H})$. If $A=T S, B=S T$, then

$$
\operatorname{dimKer}(A-\lambda)=\operatorname{dimKer}(B-\lambda), \quad \lambda \neq 0 ;
$$

moreover, if $\operatorname{Ker} S=\operatorname{Ker} T$, then $\sigma_{p}(A)=\sigma_{p}(B)$.

Proof. Suppose $\lambda \neq 0$, and $x_{i} \in \operatorname{Ker}(A-\lambda), i=1,2, \cdots, n ; x_{1}, x_{2}, \cdots, x_{n}$ are linearly independent. Then $B S x_{i}=S T S x_{i}=S A x_{i}=\lambda S x_{i}, S x_{i} \in$ 
$\operatorname{Ker}(B-\lambda), i=1,2, \cdots, n$. We claim that $S x_{1}, S x_{2}, \cdots, S x_{n}$ are linearly independent too. For otherwise, there would exist constants $\alpha_{1}, \alpha_{2}, \cdots, \alpha_{n}$, not all zero, such that $\sum_{i=1}^{n} \alpha_{i} S x_{i}=0$ and hence $\sum_{i=1}^{n} \alpha_{i} A x_{i}=\sum_{i=1}^{n} \alpha_{i} T S x_{i}=0$. Since $\sum_{i=1}^{n} \alpha_{i} A x_{i}=\lambda \sum_{i=1}^{n} \alpha_{i} x_{i}$, and $\lambda \neq 0$, therefore $\sum_{i=1}^{n} \alpha_{i} x_{i}=0$, a contradiction with the supposition that $x_{1}, x_{2}, \cdots, x_{n}$ are linearly independent. Therefore $\operatorname{dimKer}(A-\lambda) \leq \operatorname{dimKer}(B-\lambda)$. A similar argument shows that $\operatorname{dimKer}(B-\lambda) \leq$ $\operatorname{dimKer}(A-\lambda)$. It follows that $\operatorname{dimKer}(A-\lambda)=\operatorname{dimKer}(B-\lambda), \quad \lambda \neq 0$.

This equality implies $\sigma_{p}(A) \backslash\{0\}=\sigma_{p}(B) \backslash\{0\}$.

If $0 \in \sigma_{p}(A)$, then there exists $x \in \mathbf{H}, x \neq 0$ and $A x=T S x=0$. This implies $B S x=0$. If $S x \neq 0$, then $0 \in \sigma_{p}(B)$. If $S x=0$, then it follows from $\operatorname{Ker} S=\operatorname{Ker} T$ that $T x=0$ and $B x=0$; this implies $0 \in \sigma_{p}(B)$, too. Similarly $0 \in \sigma_{p}(B)$ implies $0 \in \sigma_{p}(A)$. The conclusion $\sigma_{p}(A)=\sigma_{p}(B)$ now follows.

Lemma 3. Suppose that $A \in L(\mathbf{H})$; then $\operatorname{dimKer}(A-\lambda)=\operatorname{dimKer}(\hat{A}-\lambda)$, $\operatorname{dimKer}(A-\lambda)^{*}=\operatorname{dimKer}(\hat{A}-\lambda)^{*}, \lambda \neq 0$, and $\sigma_{p}(A)=\sigma_{p}(\hat{A})$.

Proof. Since $A=U|A|=U|A|^{\frac{1}{2}}|A|^{\frac{1}{2}}, \hat{A}=|A|^{\frac{1}{2}} \cdot U|A|^{\frac{1}{2}}, A^{*}=|A|^{\frac{1}{2}} \cdot|A|^{\frac{1}{2}} U^{*},(\hat{A})^{*}=$ $|A|^{\frac{1}{2}} U^{*} \cdot|A|^{\frac{1}{2}}$ and $\operatorname{Ker}|A|^{\frac{1}{2}}=\operatorname{Ker}\left(U|A|^{\frac{1}{2}}\right)$, the conclusions follow from Lemma 2.

Lemma 4 (12]). Let $T \in L(\mathbf{H})$ be a semi-Fredholm operator. Then there exists $\delta>0$ such that $S \in L(\mathbf{H}),\|T-S\|<\delta$ implies that $S$ is semi-Fredholm and $\nu(S) \leq \nu(T), \mu(S) \leq \mu(T)$, ind $S=\operatorname{ind} T$.

Theorem 5. Let $A \in L(\mathbf{H})$. If $A_{0}$, the pure part of $A$, has no eigenvalue, then

(i) $\sigma(A)=\sigma_{p}^{0}(A) \cup\left(\bigcup_{1 \leq n \leq \infty} \psi_{0 n}(A)\right) \cup \psi_{-}^{s}(A) \cup \psi_{\infty \infty}^{s}(A) \cup \sigma_{D}(A)$,

(ii) $A$ and $\hat{A}$ have "equal spectral structure", i.e. $\sigma_{s}(A)=\sigma_{s}(\hat{A})$, where $\sigma_{s}=$ $\sigma, \sigma_{a}, \sigma_{r}, \sigma_{B}, \sigma_{w}, \sigma_{e}, \sigma_{k}, \sigma_{D}, \psi_{n}(-\infty \leq n \leq \infty), \psi_{m n}(0 \leq m, n \leq \infty)$ or $\sigma_{p}^{0}$.

Proof. Decompose $A$ into normal and pure parts: $A=N \oplus A_{0}$. If $N=W|N|$ and $A_{0}=V\left|A_{0}\right|$ are the polar decompositions of $N$ and $A_{0}$ respectively, then it is easy to derive that $W|N|=|N| W, \hat{N}=|N|^{\frac{1}{2}} W|N|^{\frac{1}{2}}=N$ and that $\hat{A}=N \oplus \hat{A}_{0}$.

Since $\sigma_{p}\left(\hat{A_{0}}\right)=\sigma_{p}\left(A_{0}\right)=\emptyset$, we have

$$
\begin{gathered}
\sigma\left(A_{0}\right)=\left(\bigcup_{1 \leq n \leq \infty} \psi_{0 n}\left(A_{0}\right)\right) \cup \sigma_{D}\left(A_{0}\right), \\
\sigma\left(\hat{A_{0}}\right)=\left(\bigcup_{1 \leq n \leq \infty} \psi_{0 n}\left(\hat{A}_{0}\right)\right) \cup \sigma_{D}\left(\hat{A_{0}}\right) .
\end{gathered}
$$

By the property of normal operators, we have

$$
\sigma(N)=\sigma_{p}^{0}(N) \cup \psi_{\infty \infty}^{s}(N) \cup \sigma_{D}(N)
$$

(1) and (3) implies that (see [9])

$$
\sigma(A)=\sigma_{p}^{0}(A) \cup\left(\bigcup_{1 \leq n \leq \infty} \psi_{0 n}(A)\right) \cup \psi_{-}^{s}(A) \cup \psi_{\infty \infty}^{s}(A) \cup \sigma_{D}(A),
$$

i.e. (i) holds.

Now let us turn to the spectral parts of $A_{0}$ and $\hat{A_{0}}$. Let $A_{0}^{o},\left(\hat{A}_{0}\right)^{o}=\hat{A}_{0}^{o}$ be the Berberian extension (see [13], Chapter I) of $A_{0}$ and $\hat{A}_{0}$ respectively; then 
$\sigma_{a}\left(A_{0}\right)=\sigma_{p}\left(A_{0}^{o}\right)$ and $\sigma_{a}\left(\hat{A_{0}}\right)=\sigma_{p}\left(\hat{A_{0}^{o}}\right)$. By Lemma $3, \sigma_{p}\left(A_{0}^{o}\right)=\sigma_{p}\left(\hat{A_{0}^{o}}\right)$; by (1), (2), $\sigma_{a}\left(A_{0}\right)=\sigma_{D}\left(A_{0}\right)$ and $\sigma_{a}\left(\hat{A}_{0}\right)=\sigma_{D}\left(\hat{A}_{0}\right)$. It follows then that

$$
\sigma_{D}\left(A_{0}\right)=\sigma_{D}\left(\hat{A_{0}}\right) \text {. }
$$

It follows from Lemma 3 and (4) that

$$
\psi_{0 n}\left(A_{0}\right) \backslash\{0\}=\psi_{0 n}\left(\hat{A}_{0}\right) \backslash\{0\}, 0 \leq n \leq \infty .
$$

Since $\psi_{0 n}\left(A_{0}\right)$ and $\psi_{0 n}\left(\hat{A_{0}}\right)$ are open sets by Lemma 4 , and $\sigma_{D}\left(A_{0}\right)=\sigma_{D}\left(\hat{A_{0}}\right)$, it is easy to derive that

$$
\psi_{0 n}\left(A_{0}\right)=\psi_{0 n}\left(\hat{A_{0}}\right), 0 \leq n \leq \infty
$$

(1) - (5) imply that

$$
\begin{gathered}
\psi_{m n}(A)=\psi_{m n}(\hat{A})=\emptyset, m>n, \\
\psi_{m n}(A)=\psi_{m m}(N) \cap \psi_{0, n-m}\left(A_{0}\right)=\psi_{m m}(N) \cap \psi_{0, n-m}\left(\hat{A_{0}}\right)=\psi_{m n}(\hat{A}), \\
0 \leq m \leq n, m<\infty . \\
\psi_{\infty \infty}(A)=\psi_{\infty \infty}^{s}(N) \backslash \sigma_{D}\left(A_{0}\right)=\psi_{\infty \infty}^{s}(N) \backslash \sigma_{D}\left(\hat{A}_{0}\right)=\psi_{\infty \infty}(\hat{A}),
\end{gathered}
$$$$
\sigma_{D}(A)=\sigma_{D}(\hat{A}),
$$

or briefly,

$$
\psi_{m n}(A)=\psi_{m n}(\hat{A}), 0 \leq m, n \leq \infty .
$$

The equalities $\sigma_{s}(A)=\sigma_{s}(\hat{A})$ in (ii) come now immediately from (6), (7) and the definitions and fundamental properties of various $\sigma_{s}$.

Theorem 6. If $A \in p-\mathbf{H}, 0<p \leq 1$, then $A$ has "equal spectral structure" (see Theorem 5) with the semi-hyponormal operator $\hat{A}$ and the hyponormal operator $\tilde{A}$.

Proof. Obvious from Lemma 1 and Theorem 5.

\section{SubdeCOMPosability AND QUASISIMILARITY}

Theorem 7. Let $T \in L(\mathbf{H}), \lambda \in \mathbf{C}$. If $\operatorname{Ker} T \subset \operatorname{Ker} T^{*}$, then

(1) $\lambda \in A(T)$ if and only if $\lambda \in A(\hat{T})$,

(2) $\lambda \in E_{2}(T)$ if and only if $\lambda \in E_{2}(\hat{T})$,

(3) $T \in(\beta)$ if and only if $\hat{T} \in(\beta)$.

Proof. Since $\operatorname{Ker} T \subset \operatorname{Ker} T^{*}$, we can write $T=\theta \oplus T_{1}$, where $\theta=\left.T\right|_{\operatorname{ker} T}, T_{1}=$ $\left.T\right|_{(\operatorname{Ker} T)^{\perp}}$. Write $\mathbf{H}_{1}=(\operatorname{Ker} T)^{\perp}$ and let $T_{1}=U\left|T_{1}\right|$ be the polar decomposition. It is clear that $\operatorname{Ker} T_{1}=\{0\}$ and $\hat{T}=\theta \oplus \hat{T}_{1}$. Thus, to prove the required result it suffices to show that $\lambda \in A\left(T_{1}\right)\left(E_{2}\left(T_{1}\right)\right)$ if and only if $\lambda \in A\left(\hat{T}_{1}\right)\left(E_{2}\left(\hat{T}_{1}\right)\right)$.

(1) Suppose that $\lambda \in A\left(T_{1}\right)$; then there exists $\delta>0$ such that for $\Omega=O\left(\lambda, \delta^{\prime}\right)$, $0<\delta^{\prime}<\delta, \alpha_{T_{1}, \Omega}$ is injective. Let $f \in \mathcal{O}\left(\Omega, \mathbf{H}_{1}\right),\left(\hat{T}_{1}-z\right) f(z)=0(z \in \Omega)$. Then $\left(T_{1}-z\right) U\left|T_{1}\right|^{\frac{1}{2}} f(z)=U\left|T_{1}\right|^{\frac{1}{2}}\left(\hat{T}_{1}-z\right) f(z)=0$. Since $\alpha_{T_{1}, \Omega}$ is injective, $U\left|T_{1}\right|^{\frac{1}{2}} f(z)=$ $0(z \in \Omega)$. It follows from $\operatorname{Ker} U\left|T_{1}\right|^{\frac{1}{2}}=\operatorname{Ker} T_{1}=\{0\}$ that $f(z)=0(z \in \Omega)$. Thus $\lambda \in A\left(\hat{T}_{1}\right)$.

The argument for the converse statement is similar. 
(2) Suppose that $\lambda \in E_{2}\left(\hat{T}_{1}\right)$; then there exists $\delta>0$ such that for $\Omega=$ $O\left(\lambda, \delta^{\prime}\right), 0<\delta^{\prime}<\delta, \alpha_{\hat{T}_{1}, \Omega}$ has closed range. Suppose that $f_{n} \in \mathcal{O}\left(\Omega, \mathbf{H}_{1}\right), n=$ $1,2, \ldots,\left(T_{1}-z\right) f_{n} \rightarrow g \in \mathcal{O}\left(\Omega, \mathbf{H}_{1}\right)$; then $\left|T_{1}\right|^{\frac{1}{2}}\left(U\left|T_{1}\right|-z\right) f_{n} \rightarrow\left|T_{1}\right|^{\frac{1}{2}} g$, i.e. $\left(\hat{T}_{1}-z\right)\left|T_{1}\right|^{\frac{1}{2}} f_{n} \rightarrow\left|T_{1}\right|^{\frac{1}{2}} g$. By the hypothesis $\lambda \in E_{2}\left(\hat{T}_{1}\right)$, there exists $f \in \mathcal{O}\left(\Omega, \mathbf{H}_{1}\right)$ such that $\left|T_{1}\right|^{\frac{1}{2}} g=\left(\hat{T}_{1}-z\right) f$. A simple calculation shows that

$$
f(z)=\frac{1}{z}\left(\hat{T}_{1} f(z)-\left|T_{1}\right|^{\frac{1}{2}} g(z)\right)=\left|T_{1}\right|^{\frac{1}{2}}\left(\frac{U\left|T_{1}\right|^{\frac{1}{2}} f(z)-g(z)}{z}\right) \quad(z \in \Omega \backslash\{0\}) .
$$

If $\lambda \neq 0$, we may assume that $0 \notin \Omega$. Let $\phi(z)=\frac{U\left|T_{1}\right|^{\frac{1}{2}} f(z)-g(z)}{z}(z \in \Omega)$. It is obvious then that $\phi \in \mathcal{O}\left(\Omega, \mathbf{H}_{1}\right)$ and $\left|T_{1}\right|^{\frac{1}{2}} g=\left(\hat{T}_{1}-z\right)\left|T_{1}\right|^{\frac{1}{2}} \phi=\left|T_{1}\right|^{\frac{1}{2}}\left(T_{1}-z\right) \phi$. But since $\operatorname{Ker}\left|T_{1}\right|^{\frac{1}{2}}=\operatorname{Ker} T_{1}=\{0\}$, hence $g=\left(T_{1}-z\right) \phi$, and so $\alpha_{T_{1}, \Omega}$ has closed range. Thus $\lambda \in E_{2}\left(T_{1}\right)$.

If $\lambda=0$, let $h(z)=U\left|T_{1}\right|^{\frac{1}{2}} f(z)-g(z)(z \in \Omega)$; then $h \in \mathcal{O}\left(\Omega, \mathbf{H}_{1}\right)$. Since $\left|T_{1}\right|^{\frac{1}{2}} g(0)=\left|T_{1}\right|^{\frac{1}{2}} U\left|T_{1}\right|^{\frac{1}{2}} f(0)$ and $\operatorname{Ker}\left|T_{1}\right|^{\frac{1}{2}}=\operatorname{Ker} T_{1}=\{0\}$, we have

$$
h(0)=U\left|T_{1}\right|^{\frac{1}{2}} f(0)-g(0)=0 .
$$

Define

$$
\phi(z)= \begin{cases}h(z) / z, & 0 \neq z \in \Omega, \\ h^{\prime}(0), & z=0 .\end{cases}
$$

It can be verified by calculation that $\phi \in \mathcal{O}\left(\Omega, \mathbf{H}_{1}\right)$. It follows from the preceding section that $g(z)=\left(T_{1}-z\right) \phi(z)(z \in \Omega \backslash\{0\})$ and this, by virtue of the analyticity of $g(z), \phi(z)$, implies that $g(z)=\left(T_{1}-z\right) \phi(z)(z \in \Omega)$, and so $\alpha_{T_{1}, \Omega}$ has closed range. Thus $0 \in E_{2}\left(T_{1}\right)$.

The converse argument is similar.

(3) The statement is obvious from $(1),(2)$ and $T \in(\beta)$ if and only if $T \in$ $(A)$ and $T \in\left(E_{2}\right)$.

Theorem 8. Suppose that $A \in p-\mathbf{H}, 0<p \leq 1$; then $A \in(\beta)$, i.e. $A$ is subdecomposable.

Proof. Suppose $A \in p-\mathbf{H}$; then $\operatorname{Ker} A \subset \operatorname{Ker} A^{*}$ and $\operatorname{Ker} \hat{A} \subset \operatorname{Ker}(\hat{A})^{*}$ by [11 Lemma 1]. Being a hyponormal operator, $\tilde{A} \in(\beta)$; consequently $\hat{A} \in(\beta)$ and hence $A \in(\beta)$ by Theorem $7,(3)$.

Lemma 9 ([10, Corollary 3]). Let $S \in L(\mathbf{H})$ be subdecomposable, $T \in L(\mathbf{H})$. If $T \stackrel{d r}{\longrightarrow} S$, then $\sigma(S) \subset \sigma(T) ;$ if $T \stackrel{d r}{\sim} S$, then $\sigma_{e}(S) \subset \sigma_{e}(T)$.

Theorem 10. Let $A \in p-\mathbf{H}, 0<p \leq 1, B \in L(\mathbf{H})$. If $B \stackrel{d r}{\longrightarrow} A$, then $\sigma(A) \subset \sigma(B)$; if $B \stackrel{d r}{\sim} A$, then $\sigma_{e}(A) \subset \sigma_{e}(B)$.

Proof. Obvious from Theorem 8 and Lemma 9.

Corollary 11. If $A$ or $A^{*} \in p-\mathbf{H}, 0<p \leq 1, B \in L(\mathbf{H}), A \stackrel{q}{\sim} B$, then $\sigma_{e}(A) \subset$ $\sigma_{e}(B), \sigma(A) \subset \sigma(B)$.

Corollary 12. If $A, B \in p-\mathbf{H}, 0<p \leq 1, A \stackrel{q}{\sim} B$, then $\sigma_{e}(A)=\sigma_{e}(B), \sigma(A)=$ $\sigma(B)$. 


\section{ACKNOWLEDGEMENT}

The authors would like to express their sincere thanks to Professor Lin Chen for his direction and help.

\section{REFERENCES}

1. A.Aluthge, On p-hyponormal operators for $0<p<1$, Integr. Equat. Oper. Th. 13 (1990), 307-315. MR 91a:47025

2. B.P.Duggal, Quasi-similarity of p-hyponormal operators, Integr. Equat. Oper. Th. 26 (1996), 338-344. MR 98g:47019

3. M.Chō, M.Itoh and S.Ōshiro, Weyl's Theorem holds for p-hyponormal operators, Glasgow M.J. 39 (1997), 217-220. MR 98e:47038

4. B.Duggal, On the spectrum of p-hyponormal operators, Acta Sci. Math. (Szeged) 63 (1997), 623-637. MR 98m:47024

5. J.Eschmeier, A decomposable Hilbert space operator which is not strongly decomposable, Integr. Equat. Oper. Th. 11 (1988), 161-172. MR 89b:47051

6. M.Putinar, Hyponormal operators are subscalar, J.operator Theory 12 (1984), 385-395. MR 85h:47027

7. J.Eschmeier and M.Putinar, Bishop's condition ( $\beta$ ) and rich extensions of linear operators, Indiana U.M.J. 37 (1988), 325-348. MR 89k:47051

8. Liming Yang, Quasisimilarity of hyponormal and subcomposable operators, J.Functional Analysis 112 (1993), 204-217. MR 94c:47033

9. C.Apostol, The correction by compact perturbation of the singular behavior of operators, Rev.Roum.Math.Pures Appl. 21 (1976), 155-175. MR 58:7180

10. Lin Chen and Yan Zikun, Bishop's property $(\beta)$ and essential spectra of quasisimilar operators, to appear in Proc. Amer. Math. Soc. CMP 98:14

11. M.Chō and T.Huruya, p-hyponormal operators for $0<p<\frac{1}{2}$, Comentationes Math. 33 (1993), 23-29. MR 95b:47021

12. T.Kato, Perturbation theory for linear operators, Berlin Heidelberg New York Tokyo, 1984. MR 96a:47025

13. D.Xia, Spectral theory of hyponormal operators, Birkhäuser Verlag, Basel, 1983. MR 87j:47036

Department of Mathematics, Fujian Normal University, Fuzhou, 350007, The PeoPLE'S RePUBlic of China

E-mail address: xhyan@fjtu.edu.cn 